\title{
HOMOLOGY INVARIANTS OF CYCLIC COVERINGS WITH APPLICATION TO LINKS
}

\author{
BY \\ Y. SHINOHARA $\left({ }^{1}\right)$ AND D. W. SUMNERS $\left({ }^{2}\right)$
}

\begin{abstract}
The main purpose of this paper is to study the homology of cyclic covering spaces of a codimension two link. The integral (rational) homology groups of an infinite cyclic cover of a finite complex can be considered as finitely generated modules over the integral (rational) group ring of the integers. We first describe the properties of the invariants of these modules for certain finite complexes related to the complementary space of links. We apply this result to the homology invariants of the infinite cyclic cover of a higher dimensional link. Further, we show that the homology invariants of the infinite cyclic cover detect geometric splittability of a link. Finally, we study the homology of finite unbranched and branched cyclic covers of a link.
\end{abstract}

1. Introduction. If $K$ is a finite simplicial complex with nonzero first Betti number, then $K$ has, among others, an infinite cyclic covering space. The integral (rational) homology groups of this cover are finitely generated modules over the integral (rational) group ring of the infinite cyclic group. Invariants of the module structure of the homology can be defined in each case, and as one would suspect, these invariants are intimately related. In $\$ 2$ we develop the general relationship between the integral and rational invariants, and study the properties of the invariants for a certain class of finite complexes related to links. Some applications to linear graphs and the homology of subgroups of the fundamental group related to the infinite cyclic covers are also given.

In $\S 3$, we define the invariants of a higher-dimensional link, and show that these invariants, except for the 1-dimensional ones, are similar to those of a higherdimensional knot.

In $\S 4$, we consider geometric splittability of a higher-dimensional link, and give some examples of nonsplittable links which are detectable by the homology invariants.

Finally, in $\S 5$, we study the homology groups of the $k$-fold unbranched and branched cyclic covers of links, particularly the case of $q$-simple links.

Received by the editors December 21, 1970.

AMS 1970 subject classifications. Primary 55A10, 55A25, 57C45.

$K e y$ words and phrases. Infinite cyclic cover, $k$-fold unbranched cyclic cover, $k$-fold branched cyclic cover, integral invariant, rational invariant, linear graph in $S^{3}, n$-link of multiplicity $\mu$, geometrically $k$-splittable link, completely splittable link, nonsplittable link, null-cobordant link, $q$-simple link.

(1) A postdoctoral fellow of the National Research Council of Canada (Grant A 4034).

$\left(^{2}\right)$ Research partially supported by NSF GP-11943.

Copyright $(C)$ 1972, American Mathematical Society 
2. Polynomial invariants of infinite cyclic coverings. Let $K$ be an $(n+1)-$ dimensional finite simplicial complex with homology groups

$$
\begin{aligned}
H_{i}(K ; Z) & =Z, & & i=0, \\
& =\alpha Z, & & i=1, \\
& =\beta Z, & & i=n+1, \\
& =0, & & \text { otherwise, }
\end{aligned}
$$

where $\alpha Z(\beta Z)$ denotes the direct sum of $\alpha(\beta)$ copies of $Z$. Let $\widetilde{K}$ be any infinite cyclic covering space of $K$ with $J(t)$ (the infinite cyclic multiplicative group generated by $t$ ) as the group of covering translations. Let $\Lambda$ denote the integral group ring of $J(t)$ and $\Gamma=\Lambda \otimes_{z} Q$ the rational group ring of $J(t) . \Lambda$ is a Noetherian unique factorization domain and $\Gamma$ is a principal ideal domain.

For all $q$, the integral chain groups $C_{q}(\tilde{K} ; Z)$ are finitely-generated $\Lambda$-modules, with generators in 1-1 correspondence with the $q$-simplexes of $K$. Since $\Lambda$ is Noetherian, then $H_{q}(\tilde{K} ; Z)$ is a finitely-generated $\Lambda$-module. Likewise, $H_{q}(\tilde{K} ; Q)$ $\cong H_{q}(\tilde{K} ; Z) \otimes_{z} Q$ is a finitely generated $\Gamma$-module. The integral (rational) invariants are defined by using the integral (rational) homology of $\tilde{K}$. They lie in $\Lambda(\Gamma)$, and are therefore polynomials in positive and negative powers of the variable $t$. The development of the rational invariants follows closely that of $\mathrm{J}$. Levine for knots [13].

Definition: Presentation matrix of a module. Consider an abelian group $A$ which is finitely generated as a $\Lambda(\Gamma)$-module. An $m \times n$ matrix $M=\left(m_{i j}(t)\right)$ with entries in $\Lambda(\Gamma)$ is said to present $A$ as a module if there exists an exact sequence of $\Lambda(\Gamma)$-modules

$$
F_{2} \stackrel{d}{\longrightarrow} F_{1} \longrightarrow A \longrightarrow 0
$$

where $F_{1}$ and $F_{2}$ are free on the entries $\left(x_{1}, \ldots, x_{n}\right)$ and $\left(r_{1}, \ldots, r_{m}\right)$ respectively, and $d\left(r_{i}\right)=\sum_{j=1}^{n} m_{i j}(t) x_{j}$.

Definition: Elementary ideals of a matrix. Let $M$ be an $(m \times n)$ matrix with entries in $\Lambda(\Gamma)$, and $k$ an integer. The $k$ th elementary ideal $\varepsilon_{k}$ of $M$ is the ideal in $\Lambda(\Gamma)$ generated by the determinants of the $(n-k+1) \times(n-k+1)$ submatrices of $M$, with the conventions

(i) $(n-k+1)<1$ then $\varepsilon_{k}=\Lambda(\Gamma)$,

(ii) $(n-k+1)>m$ then $\varepsilon_{k}=0$.

If the matrix $M$ presents $A$ as a $\Lambda(\Gamma)$-module, then it is well known that the elementary ideals of $M$ are invariant of $\Lambda(\Gamma)$-isomorphism type of $A$. (See [22].) Since $\Lambda$ is Noetherian and $H_{q}(\widetilde{K} ; Z)$ is finitely generated, then there exists a presentation for $H_{q}(\tilde{K} ; Z)$ as a $\Lambda$-module, and likewise $H_{q}(\tilde{K} ; Q)$ as a $\Gamma$-module.

Let $M_{q}=\left(m_{i j}(t)\right)$ be a presentation matrix for $H_{q}(\tilde{K} ; Z)$. Since $-\otimes_{z} Q$ is a rightexact functor, we can take $M_{q}^{\prime}=\left(m_{i j}(t) \otimes 1\right)$ to be a presentation matrix for $H_{q}(\tilde{K} ; Q)$ as a $\Gamma$-module. 
Definition I. The $k$ th integral invariant of $\tilde{K}$ in dimension $q, \Delta_{k}^{q}(t) \in \Lambda$, is the generator of the smallest principal $\Lambda$-ideal containing $\varepsilon_{k}^{q}$, the $k$ th elementary ideal of $M_{q}$. This generator is defined up to units in $\Lambda$, and for each integer $q$ there exists $k_{q}$ an integer such that $\Delta_{i}^{q}(t)=1$ for $i>k_{q}$. In classical knot theory, the integral invariants of the knot complement are called the Alexander polynomials of the knot [7, pp. 119].

Definition II. The $k$ th rational invariant of $\tilde{K}$ in dimension $q, \Delta_{k}^{q^{\prime}}(t) \in \Gamma$, is the generator of $\varepsilon_{k}^{q^{\prime}}$, the $k$ th elementary ideal of $M_{q}^{\prime} \cdot \varepsilon_{k}^{q \prime}$ is a principal ideal because $\Gamma$ is a PID.

Definition. $p(t) \in \Lambda$ is primitive if all its coefficients are relatively prime.

Definition. $p(t), q(t) \in \Lambda(\Gamma)$ are associate in $\Lambda(\Gamma)$ if there exists a unit $h(t)$ $\in \Lambda(\Gamma)$ such that $p(t)=h(t) q(t)$.

Following Levine [13], we have the following easily-proved lemma:

Lemma 2.1. Let $\lambda, \mu \in \Lambda$ and $\lambda$ primitive. Then $\lambda \mid \mu$ in $\Lambda$ if and only if $\lambda \mid \mu$ in $\Gamma$.

Hence, it is clear that every associate class in $\Gamma$ contains a primitive element of $\Lambda$, unique up to associate class in $\Lambda$. Since the generator of an ideal in $\Gamma$ is defined only up to associate class in $\Gamma$, we can take the rational invariants of $\tilde{K}$ to be primitive elements of $\Lambda$.

As one might expect, the integral invariants of $\tilde{K}$ and the rational invariants of $\tilde{K}$ are intimately related. In fact, we have the following

LemmA 2.2. $\Delta_{k}^{q \prime}=r \Delta_{k}^{q}$ where $0 \neq r \in Q$ is the unique rational such that $r \Delta_{k}^{q}$ is primitive. (If $\Delta_{k}^{q}=0$, take $r=1$.)

Proof. See also Levine [11] and Neuwirth [17]. Regard $\Lambda$ as embedded in $\Gamma$ in the canonical way, that is, identify $\Lambda$ with $\Lambda \otimes 1$ in $\Gamma=\Lambda \otimes_{z} Q$. If $\lambda \in \Lambda$, let $[\lambda]$ denote the $\Lambda$-ideal generated by $\lambda$, and $(\lambda)$ the $\Gamma$-ideal generated by $\lambda$. Let $\varepsilon$ be a $\Lambda$-ideal. $\varepsilon$ determines a $\Gamma$-ideal $\varepsilon^{\prime}=\left(\lambda^{*}\right), \lambda^{*}$ the primitive generator of $\varepsilon^{\prime}$. Let $[\lambda]$ be the smallest principal ideal containing $\varepsilon$, and $r \in Q$ be chosen such that $r \lambda$ is primitive. It suffices to prove that $r \lambda=\lambda^{*}$ (up to associate class in $\Lambda$ ). Since $\varepsilon \subset[\lambda]$, $\varepsilon^{\prime}=\left(\lambda^{*}\right) \subset(\lambda)=(r \lambda)$. On the other hand, $\varepsilon \subset \varepsilon^{\prime} \cap \Lambda=\left[\lambda^{*}\right]$, so $[\lambda] \subset\left[\lambda^{*}\right]$, hence $(r \lambda)$ $=(\lambda) \subset\left(\lambda^{*}\right)$ and $\lambda^{*}=r \lambda$.

Hence if $\Delta_{k}^{q}$ is primitive (as is the case when $\Delta_{k}^{q}(1)= \pm 1$ ), then $\Delta_{k}^{q \prime}=\Delta_{k}^{q}$.

We will need the following technical lemma:

Lemma 2.3. If $R$ is a Noetherian ring, $A$ is a f.g. $R$-module and $\phi: A \rightarrow A$ is an epimorphism then $\phi$ is an isomorphism.

Proof. Let $K_{n}=\operatorname{Ker} \phi^{n}$. Then $K_{1} \subset K_{2} \subset \cdots$ and since $R$ is Noetherian and $A$ is f.g., this ascending chain of submodules of $A$ has a maximal element, say $K_{q}$. So $K_{q+i}=K_{q} \forall i \geqq 0$, hence if $\phi^{q}(x) \neq 0$ for some $x \in A$ then $\phi^{q+i}(x) \neq 0 \forall i \geqq 0$. This means that $\operatorname{Ker} \phi=K_{1}=0$, because if $x \in K_{1}$ then $\exists y \in A$ such that $\phi^{q}(y)=x$ since $\phi^{q}$ is an epimorphism. But $0=\phi(x)=\phi^{q+1}(y)$ so $0=\phi^{q}(y)=x$. 
We now have the following theorem describing the properties of the integral invariants:

THEOREM 2.4. $K^{n+1}$ as above, $n \geqq 2$ and $\left\{\Delta_{i}^{q}\right\}$ the integral invariants of $\tilde{K}$. Then

(i)

(ii)

$$
\begin{aligned}
& \Delta_{i+1}^{q} \mid \Delta_{i}^{q} \text { in } \Lambda, \\
& \Delta_{i}^{q}(1)=0, \quad q=0, \quad i=1, \\
& =0, \quad q=1, \quad i \leqq \alpha-1, \\
& =0, \quad q=n+1, \quad i \leqq \beta, \\
& = \pm 1 \text {, otherwise. }
\end{aligned}
$$

Proof. Adapting an argument of Milnor [15], consider the short exact sequence of chain complexes with integral coefficients

$$
0 \longrightarrow C_{*}(\tilde{K}) \stackrel{(t-1)}{\longrightarrow} C_{*}(\tilde{K}) \longrightarrow C_{*}(K) \longrightarrow 0 .
$$

This induces the long exact sequence of homology

$$
\longrightarrow H_{i}(\tilde{K}) \stackrel{(t-1)}{\longrightarrow} H_{i}(\tilde{K}) \longrightarrow H_{i}(K) \stackrel{\partial}{\longrightarrow} H_{i-1}(\tilde{K}) \longrightarrow
$$

Think of $\boldsymbol{Z}$ as a $\Lambda$-module via the augmentation map

$$
\stackrel{\boldsymbol{\varepsilon}}{\longrightarrow} \boldsymbol{Z}
$$

If the matrix $M_{q}(t)=\left(m_{i j}(t)\right)$ presents $H_{q}(\tilde{K})$ as a $\Lambda$-module, then $M_{q}(1)$ presents $H_{q}(\tilde{K}) \otimes_{\Lambda} Z$ as an abelian group. Moreover, $\left\{\Delta_{i}^{q}(1)\right\}$ are invariants of $H_{q}(\widetilde{K}) \otimes_{\Lambda} Z$ as a $Z$-module, and $H_{q}(\tilde{K}) \otimes_{\Lambda} Z$ is isomorphic to the cokernel of the homomorphism $H_{q}(\tilde{K}) \stackrel{(t-1)}{\longrightarrow} H_{q}(\widetilde{K})$. Since $H_{*}(K)$ satisfies (2.1), (2.2) yields immediately that $H_{q}(\widetilde{K}) \otimes_{\Lambda} Z=0,2 \leqq q \leqq n-1(n \geqq 3)$. At the top end of the exact sequence we have

$$
\begin{aligned}
0 \longrightarrow H_{n+1}(\tilde{K}) \stackrel{(t-1)}{\longrightarrow} H_{n+1}(\tilde{K}) & \longrightarrow H_{n+1}(K) \\
& \longrightarrow H_{n}(\tilde{K}) \stackrel{(t-1)}{\longrightarrow} H_{n}(\tilde{K}) \longrightarrow 0 .
\end{aligned}
$$

By Lemma 2.3, $(t-1): H_{n}(\widetilde{K}) \rightarrow H_{n}(\tilde{K})$ is an isomorphism, so $H_{n+1}(\tilde{K}) \otimes_{\Lambda} Z$ $\cong H_{n+1}(K)=\beta Z$. At the bottom end of the exact sequence we have

$$
\begin{aligned}
& 0 \longrightarrow H_{1}(\tilde{K}) \stackrel{(t-1)}{\longrightarrow} H_{1}(\tilde{K}) \longrightarrow H_{1}(K) \\
& \text { II? } \\
& \alpha \boldsymbol{Z}
\end{aligned}
$$

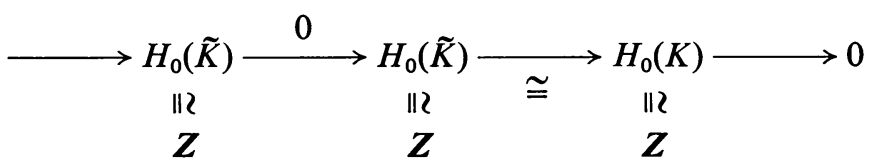


hence $H_{1}(\widetilde{K}) \otimes_{\Lambda} Z \cong(\alpha-1) Z$. Now since $\tilde{K}$ is connected, $\Delta_{1}^{0}(t)=(t-1)$. This completes the proof of Theorem 2.4.

There is a better way of thinking of the rational invariants of $\tilde{K}$. $\Gamma$ is a principal ideal domain, so by the structure theorem for finitely-generated modules over a PID, $H_{q}(\tilde{K} ; Q) \cong{ }_{\Gamma} F_{q} \oplus T_{q}$ where $F_{q}$ is the direct sum of copies of $\Gamma$, and $T_{q}$ is the direct sum of cyclic modules

$$
T_{q} \cong \Gamma / \lambda_{1}^{q} \oplus \cdots \oplus \Gamma / \lambda_{m}^{q}, \quad \lambda_{i}^{q} \in \Lambda, \lambda_{i+1}^{q} \mid \lambda_{i}^{q} \text { in } \Lambda \forall i
$$

Here $\Gamma / \lambda$ denotes the cokernel of the homomorphism

$$
\begin{aligned}
& \Gamma \longrightarrow \Gamma \\
& 1 \longmapsto \lambda
\end{aligned} \quad \text { (see Levine [13]). }
$$

That is, if the $\Gamma$-rank of $F_{q}$ is $r_{q}$, then $H_{q}(\tilde{K} ; Q)$ is presented as a $\Gamma$-module by the diagonal matrix

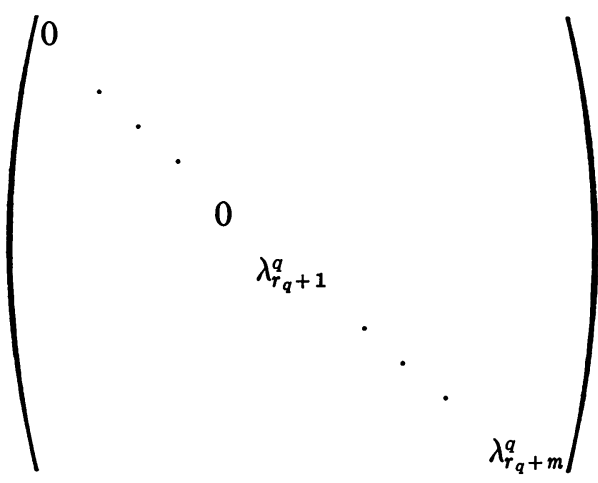

and the rational invariants are given by the formula

$$
\begin{aligned}
\Delta_{j}^{q^{\prime}} & =0, & & 1 \leqq j \leqq r_{q}, \\
& =\prod_{i=j}^{r_{q}+m} \lambda_{i}^{q}, & & r_{q}+1 \leqq j \leqq r_{q}+m, \\
& =1, & & r_{q}+m+1 \leqq j .
\end{aligned}
$$

CoROLlaRY 2.5. $K$ as above and $n \geqq 2$, then $H_{q}(\tilde{K} ; Q) \cong F_{q} \oplus T_{q}$ where $r_{q}(\Gamma$-rank of $H_{q}$ )

$$
\begin{aligned}
& =\alpha-1, \quad q=1, \\
& =\beta, \quad q=n+1 \text {, } \\
& =0, \quad \text { otherwise. }
\end{aligned}
$$

Moreover, the integral invariants $\Delta_{j}^{q}(t)=0$ for $q=1,1 \leqq j \leqq \alpha-1$ and $q=n+1$, 
$1 \leqq j \leqq \beta$, and $T_{q}$ is the torsion module presented by the diagonal matrix $\left(\lambda_{i}^{q}\right)$ with $\lambda_{i}^{q} \in \Lambda, \lambda_{i+1}^{a} \mid \lambda_{i}^{q}$ in $\Lambda$, and

$$
\begin{aligned}
\lambda_{i}^{q}(1) & =0, & & q=0, i=1, \\
& = \pm 1, & & \text { otherwise. }
\end{aligned}
$$

Theorem 2.4 and Corollary 2.5 avoid the case where $n=1$. We will now study the rational invariants in this case.

Let $r_{i}=\Gamma$-rank of $H_{i}(\tilde{K} ; Q), i=1,2$. We have as in Theorem 2.4 the exact sequence

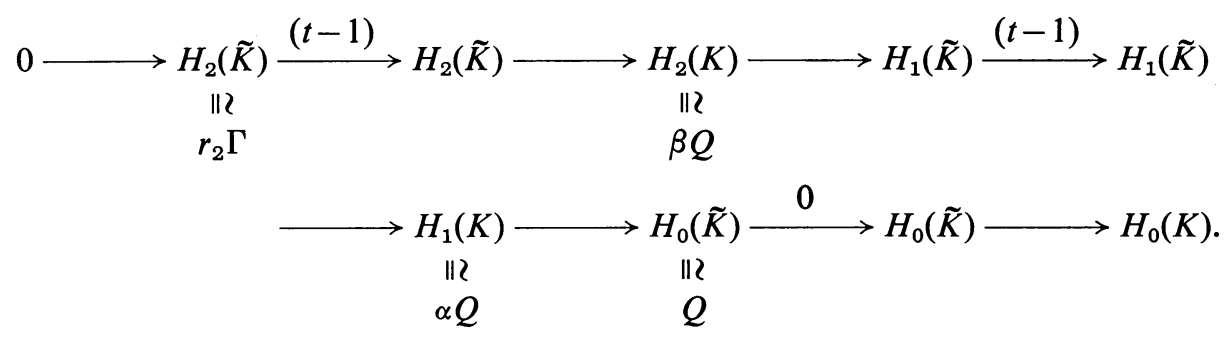

This reduces to

$$
\begin{aligned}
0 \longrightarrow & \left.\longrightarrow r_{2}\right) Q \longrightarrow H_{1}(\tilde{K}) \stackrel{(t-1)}{\longrightarrow} H_{1}(\tilde{K}) \\
& \longrightarrow(\alpha-1) Q \longrightarrow 0 .
\end{aligned}
$$

Let $H_{1}(\widetilde{K} ; Q) \cong_{\Gamma} F_{1} \oplus T_{1}$ as before. Consider the prime-power-order decomposition for the torsion module $T_{1}$. That is,

$$
T_{1} \cong \Gamma /(t-1)^{p_{1}} \oplus \Gamma /(t-1)^{p_{2}} \oplus \cdots \oplus \Gamma /(t-1)^{p_{k}} \oplus T_{1}^{\prime}
$$

where the prime order $(t-1)$ does not appear in $T_{1}^{\prime}$. The homomorphism $(t-1)$ respects any $\Gamma$-splitting, and is injective on $F_{1}$ and an isomorphism on $T_{1}^{\prime}$. Also

$$
\begin{aligned}
0 \longrightarrow \Gamma /(t-1) \longrightarrow \Gamma /(t-1)^{p_{i}} \stackrel{(t-1)}{\longrightarrow} \Gamma /(t-1)^{p_{i}} \\
\longrightarrow \Gamma /(t-1) \longrightarrow 0
\end{aligned}
$$

is exact for all $1 \leqq i \leqq k$. Hence (2.3) yields the equations $k=\beta-r_{2}$ and $k+r_{1}=\alpha-1$, so $\beta-r_{2}=\alpha-1-r_{1}$. In the above $k$ represents the number of times a power of $(t-1)$ appears in the prime power decomposition of $T_{1}$. Hence we have $\chi(K)=r_{2}-r_{1}$, where $\chi(K)$ denotes the Euler characteristic of $K$.

THEOREM 2.6. Let $K^{2}$ be as above. Then

(i) $\Delta_{i}^{1 \prime}=0$ for $i \leqq \alpha-\beta-1$, if $\alpha-\beta-1 \geqq 1$.

(ii) $\Delta_{k}^{1 \prime}$ is the first nonvanishing rational invariant of $\tilde{K}$ (i.e. $\Delta_{j}^{1}=0,1 \leqq j \leqq k-1$, $\Delta_{k}^{\prime \prime} \neq 0$ ) if and only if $r_{2}=\beta-\alpha+k$. 
Definition. Let $P$ be a finite 1-dimensional subcomplex of $S^{3}$, such that $H_{1}(P ; Z) \neq 0$. Then $P$ is called a linear graph in $S^{3}$.

Let $P$ be a linear graph in $S^{3}$, and $X=S^{3}-P, G=\Pi_{1}(X)$, and $G^{*}=\operatorname{Ker} \varphi$ where $\varphi: G \rightarrow J(t)$ is some epimorphism. Let $\tilde{X}$ be the infinite cyclic cover of $X$ corresponding to $G^{*}$.

THEOREM 2.7. Let $\alpha_{0}$ be the number of components of $P$ and $\beta_{0}$ be the 1-dimensional Betti number of $P$. Then

(i) $\Delta_{i}^{1}(1)=0, i \leqq \beta_{0}-1,= \pm 1, i \geqq \beta_{0}$,

(ii) $\Delta_{i}^{1}(t)=0, i \leqq \beta_{0}-\alpha_{0}$ if $\left(\beta_{0}-\alpha_{0}\right) \geqq 1$,

(iii) $\Delta_{\beta_{0}-\alpha_{0}+m}^{1}(t)$ is the first nonvanishing integral invariant of $\tilde{X}$ if and only if $\mathrm{H}_{2}(\tilde{X} ; Q)$ is a free $\Gamma$-module of rank $m-1$.

REMARK. The properties (i) and (ii) are similar to Kinoshita's [10].

Proof. By Alexander duality, $H_{*}(X ; Z)$ satisfies the condition (2.1) for $\alpha=\beta_{0}$ and $\beta=\alpha_{0}-1$. Further, $X$ has a 2-dimensional subcomplex as its deformation retract. Hence (ii) and (iii) are immediate consequences of Lemma 2.2 and Theorem 2.6.

As in Theorem 2.4, we have an exact sequence

$$
H_{1}(\tilde{X} ; Z) \stackrel{(t-1)}{\longrightarrow} H_{1}(\tilde{X} ; Z) \longrightarrow\left(\beta_{0}-1\right) Z \longrightarrow 0,
$$

so Coker $(t-1) \cong\left(\beta_{0}-1\right) Z$. This yields (i).

Definition. A linear graph $P$ in $S^{3}$ is said to be nonsplittable if given any embedded $S^{2}$ in $S^{3}$ such that $S^{2} \cap P=\varnothing$, then $P$ is contained entirely within one of the complementary domains.

COROLlaRy 2.8. Let $P$ be a nonsplittable linear graph in $S^{3}$. Then $H_{2}\left(G^{*} ; Z\right)=0$ iff $\Delta_{\beta_{0}-\alpha_{0}+1}^{1} \neq 0$.

Proof. The proof follows [20]. By Theorem 2.7(iii) we have $H_{2}(\tilde{X} ; Q)=0$. Since $\tilde{X}$ is 2-dimensional, this means that $H_{2}(\tilde{X} ; Z)=0$. Now $P$ is nonsplittable, so by [18] $X$ is aspherical and $\tilde{X}=K\left(G^{*}, 1\right)$ the Eilenberg-Mac Lane space. Hence $H_{2}\left(G^{*} ; Z\right) \cong H_{2}(K(G, 1) ; Z)=0$.

This corollary is analogous to a result by Crowell and Cochran [6]. The splittable case will be studied in $\S 4$.

Let $P$ be any tamely embedded linear graph as before, $G=\Pi_{1}\left(S^{3}-P\right)$ and $\varphi_{1}: G \rightarrow Z$ an epimorphism. Let $\tilde{X}_{1}$ be the infinite cyclic covering space associated with Kernel $\varphi_{1}$ and $\left\{\Delta_{i}^{1}\right\}$ the integral invariants of $\tilde{X}_{1}$. Let $X=S^{3}-P$ and $G^{\prime}=[G, G]$.

THEOREM 2.9. Let $\tilde{X}$ be the universal abelian covering of $X$. If $\Delta_{\beta_{0}-\alpha_{0}+1}^{1} \neq 0$, then $H_{2}(\tilde{X} ; Z) \cong 0$.

Proof. By Theorem 2.7 we have $H_{2}\left(\tilde{X}_{1} ; Z\right) \cong 0$. The epimorphism $\varphi_{1}: G \rightarrow Z$ factors through $H_{1}(X ; Z) \cong G / G^{\prime} \cong \beta_{0} Z$. Hence $G^{\prime}$ is a normal subgroup of $G_{1}$ $=\operatorname{Kernel} \varphi_{1}$, and $G^{\prime} \supset\left[G_{1}, G_{1}\right]$. 
Now $\left(G / G^{\prime}\right) /\left(G_{1} / G^{\prime}\right) \cong G / G_{1} \cong Z$, hence $0 \rightarrow G_{1} / G^{\prime} \rightarrow \beta_{0} Z \rightarrow Z \rightarrow 0$ is split exact and $G_{1} / G^{\prime} \cong\left(\beta_{0}-1\right) Z$. Let $\varphi_{2}: G_{1} \rightarrow Z$ be the epimorphism indicated below:

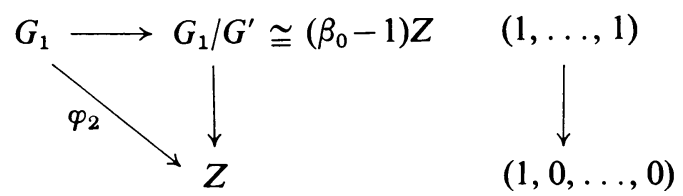

Let $G_{2}$ be the kernel of $\varphi_{2}: G_{1} \rightarrow Z$. As before, we have $G_{2} / G^{\prime} \cong\left(\beta_{0}-2\right) Z$. Let $\tilde{X}_{2}$ be the infinite cyclic covering space of $\tilde{X}_{1}$ associated with Kernel $\varphi_{2}$. Let $J(t)$ denote the infinite cyclic group of covering translations of $\tilde{X}_{2}$. As before, we have the long exact homology sequence

$$
0 \longrightarrow H_{2}\left(\tilde{X}_{2} ; Z\right) \stackrel{t-1}{\cong} H_{2}\left(\tilde{X}_{2} ; Z\right) \longrightarrow 0 .
$$

Since $\tilde{X}_{2}$ is 2 -dimensional, then as before $H_{2}\left(\tilde{X}_{2} ; Z\right) \cong 0$. The process can be repeated $\beta_{0}$ times, obtaining a stack of covering spaces

$$
\tilde{X}_{\beta_{0}} \rightarrow \cdots \rightarrow \tilde{X}_{2} \rightarrow \tilde{X}_{1} \rightarrow X
$$

each $\tilde{X}_{i}$ being an infinite cyclic covering space of $\tilde{X}_{i-1}$, and $\tilde{X}_{\beta_{0}}$ being the universal abelian covering of $X$. Hence $H_{2}(\tilde{X} ; Z) \cong 0$.

Corollary 2.10. If $P$ is a nonsplittable linear graph in $S^{3}$ and $\Delta_{\beta_{0}-\alpha_{0}+1}^{1} \neq 0$, where $\Delta_{\beta_{0}-\alpha_{0}+1}^{1}$ is the invariant associated with some infinite cyclic cover of $X$, then $H_{2}\left(G^{\prime} ; Z\right)=0$.

Proof. Since $X$ is aspherical, then as before $\tilde{X}=K\left(G^{\prime}, 1\right)$ and the result follows from Theorem 2.9.

\section{Polynomial invariants of higher dimensional links.}

Definition. An $n$-link $L=K_{1} \cup \cdots \cup K_{\mu}$ of multiplicity $\mu$ is the disjoint union of $\mu$ oriented and smoothly embedded $n$-spheres $K_{i}$ in $S^{n+2}$. Two $n$-links $L$ and $L^{\prime}$ of multiplicity $\mu$ belong to the same link type if there exists an orientation preserving homeomorphism $f$ of $S^{n+2}$ onto itself such that $f\left(K_{i}\right)=K_{i}^{\prime}$ and $f \mid K_{i}$ is orientation preserving, $i=1,2, \ldots, \mu$.

Let $X$ be the complement of an open tubular neighborhood of $L$ in $S^{n+2}$ and $\tilde{X}$ the covering space of $X$ belonging to $\operatorname{Ker} \varphi$, where $\varphi: \Pi_{1}(X) \rightarrow J(t)$ is an epimorphism given by

$$
\varphi(g)=t^{\operatorname{link}(g, L)} \text { for } g \in \Pi_{1}(X),
$$

link $(g, L)=\sum_{i}$ link $\left(g, K_{i}\right) . X$ is a finite complex and $\tilde{X}$ is an infinite cyclic cover of $X$. Hence, as in $\S 2$, the integral invariants $\left\{\Delta_{i}^{q}(t)\right\}$ and the rational invariants 
$\left\{\Delta_{i}^{q^{\prime}}(t)\right\}$ of $\tilde{X}$ are well defined. It is easily seen that these are link type invariants. If $n=1, \Delta_{1}^{1}(t)$ is known as the reduced Alexander polynomial of $L$ and its properties have been studied. For the case $n \geqq 2$, we have the following theorem:

THEOREM 3.1. Let $L$ be an $n$-link of multiplicity $\mu$ and $n \geqq 2$. Then

$$
\begin{aligned}
& \Delta_{i+1}^{q}(t) \mid \Delta_{i}^{q}(t) \text { in } \Lambda \text {, } \\
& \Delta_{i}^{q}(1)=0, \quad q=0, i=1, \\
& =0, \quad q=1, i \leqq \mu-1, \\
& =0, \quad q=n+1, i \leqq \mu-1 \text {, } \\
& = \pm 1, \quad \text { otherwise, } \\
& \Delta_{i}^{1}(t)=0, \quad i<\mu, \\
& =\Delta_{i-\mu+1}^{n}\left(t^{-1}\right), \quad i \geqq \mu, \\
& \Delta_{i}^{q}(t)=\Delta_{i}^{n+1-q}\left(t^{-1}\right), \quad 2 \leqq q \leqq n-1 .
\end{aligned}
$$

Proof. $X$ has the same homotopy type as an $(n+1)$-dimensional complex, and by Alexander duality it satisfies the condition (2.1) for $\alpha=\mu$ and $\beta=\mu-1$. Hence Theorem 2.4 yields (i) and (ii).

Now $\partial \tilde{X}$ is the disjoint union of $\mu$ copies of $S^{n} \times R^{1}$. Hence it follows easily that $H_{i}(\tilde{X}) \cong H_{i}(\tilde{X}, \partial \tilde{X})$ for $2 \leqq i \leqq n-1$. Further, $H_{n}(\partial \tilde{X})$ is the direct sum of $\mu$ copies of $\Lambda /(t-1)$ and $\Delta_{1}^{n}(1)= \pm 1$. Hence, in the exact sequence of $\Lambda$-modules

$$
\cdots \longrightarrow H_{n}(\partial \tilde{X}) \stackrel{i_{*}}{\longrightarrow} H_{n}(\tilde{X}) \longrightarrow H_{n}(\tilde{X}, \partial \tilde{X}) \longrightarrow 0,
$$

$i_{*}$ is trivial, which implies $H_{n}(\tilde{X}) \cong{ }_{\Lambda} H_{n}(\tilde{X}, \partial \tilde{X})$.

Theorem 2.4 and Corollary 2.5 show that $H_{i}(\tilde{X})$ is a torsion $\Lambda$-module for $2 \leqq i \leqq n$ and $\mu-1$ is the maximal number of linearly independent elements in the $\Lambda$-module $H_{1}(\tilde{X})$. Hence Corollary 4.8 and Lemma 4.10 of [4] yield (iii).

Definition. An $n$-link $L=K_{1} \cup \cdots \cup K_{\mu}$ of multiplicity $\mu$ is called nullcobordant if there exist $\mu$ mutually disjoint $(n+1)$-balls $D_{1}^{n+1}, \ldots, D_{\mu}^{n+1}$ in $D^{n+3}$ with $\partial D_{i}^{n+1}=K_{i}, i=1,2, \ldots, \mu$, where $\partial D^{n+3}=S^{n+2}$.

K. Murasugi showed that for a null-cobordant 1-link of multiplicity $\mu$

$$
\begin{aligned}
\Delta_{i}^{1}(t) & =0, & & i<\mu, \\
& =f(t) f\left(t^{-1}\right), & & i=\mu,
\end{aligned}
$$

where $f(t) \in \Lambda$ and $f(1)= \pm 1$ [16]. For higher-dimensional links we have

THEOREM 3.2. If $L$ is a null-cobordant $(2 m-1)$-link of multiplicity $\mu$ and $m \geqq 2$, then $\Delta_{1}^{m}(t)=f(t) f\left(t^{-1}\right)$ for some $f(t) \in \Lambda$ with $f(1)= \pm 1$.

Proof. The proof is analogous to the case of null-cobordant knots [12]. By using the Thom-Pontrjagin construction as in Levine [14], one can show that every 
$n$-link bounds a smooth connected $(n+1)$-manifold $V$ in $S^{n+2}$. If $n=2 m-1$, we define a Seifert matrix $A$ of $V$ as in [12]. The argument in $\$ 2$ of [13] together with the fact that $H_{q}(\tilde{X} ; Q)$ is a torsion $\Gamma$-module for $2 \leqq q \leqq n$ shows that $t A+(-1)^{m} A^{\prime}$ is a presentation matrix for $H_{m}(\tilde{X} ; Q)$. Finally, by modifying the proof of Lemma 2 of [12] it can be shown that if $L$ is null-cobordant, then $A$ is null-cobordant in the sense of [12], which yields the theorem.

\section{$\dot{4}$. Detecting geometric linking.}

Definition. An $n$-link $L=K_{1} \cup \cdots \cup K_{\mu}$ of multiplicity $\mu$ is said to be geometrically $k$-splittable $(0 \leqq k \leqq \mu-1)$ if $\exists B=B_{1}^{n+2} \cup \cdots \cup B_{k}^{n+2}$, a collection of $k$ mutually disjoint sub-balls of $S^{n+2}$ satisfying

(i) $B_{i}^{n+2} \cap L \neq \varnothing 1 \leqq i \leqq k$,

(ii) $\partial B \cap L=\varnothing$,

(iii) $S^{n+2}-B \cap L \neq \varnothing$,

(iv) $k$ is maximal with respect to the above properties.

If $k=\mu-1$, we say that $L$ is completely splittable, and if $k=0$ we say that $L$ is nonsplittable. If $L=K_{1} \cup \cdots \cup K_{\mu}$ is $k$-splittable, then we say that

$$
\begin{aligned}
L_{i} & =L \cap B_{i}^{n+2}, \quad 1 \leqq i \leqq k, \\
L_{k+1} & =L \cap\left(S^{n+2}-B\right)
\end{aligned}
$$

are the nonsplittable components of $L$.

Suppose that $L=K_{1} \cup K_{2}$ is an $n$-link of multiplicity 2. Let $X=S^{n+2}-L$, and $\tilde{X}$ be the infinite cyclic covering space of $X$ as in $\S 3$. Let $X_{i}=S^{n+2}-K_{i}$ and $\tilde{X}_{i}$ be the infinite cyclic cover of $X_{i}, i=1,2$.

THEOREM 4.1. If $L$ as above is completely splittable, then

$$
\begin{aligned}
H_{i}(\tilde{X} ; Z) & \cong{ }_{\Lambda} H_{i}\left(\tilde{X}_{1} ; Z\right) \oplus H_{i}\left(\tilde{X}_{2} ; Z\right), \quad 2 \leqq i \leqq n, \\
H_{1}(\tilde{X} ; Z) & \cong{ }_{\Lambda} H_{1}\left(\tilde{X}_{1} ; Z\right) \oplus H_{1}\left(\tilde{X}_{2} ; Z\right) \oplus \Lambda, \\
H_{n+1}(\tilde{X} ; Z) & \cong{ }_{\Lambda} H_{n+1}\left(\tilde{X}_{1} ; Z\right) \oplus H_{n+1}\left(\tilde{X}_{2} ; Z\right) \oplus \Lambda \cong \Lambda .
\end{aligned}
$$

Proof. Let $T_{i}$ be the closed tubular neighborhood of $K_{i}$ in $S^{n+2}, i=1,2$. Then $S^{n+2}-K_{2} \simeq X_{2} \vee B_{1}^{n+2}$ where $B_{1}^{n+2}$ is the $n+2$-ball given by the splitting, $B_{1}^{n+2} \supset K_{1}$. That is, we can isolate any $B^{n+2}$ missing $K_{2}$ in $S^{n+2}$ in a wedge product decomposition for $X_{2}$. Let $C$ be a collar on $\partial B_{1}^{n+2}$ contained in $S^{n+2}-B_{1}^{n+2}$ and missing $T_{2}$. Let $\partial C$ be the exterior boundary of $C$. Connect $\partial T_{2}$ to $\partial C$ by an arc whose interior is contained in $S^{n+2}-\left(B_{1}^{n+2} \cup C \cup T_{2}\right)$. Collapse away from $\partial T_{2}$ in a tubular neighborhood of the arc until $\partial C$ is reached. This frees a top-dimensional disc on $\partial C$, and $C$ can be then collapsed to an arc connecting $B_{1}^{n+1}$ to $\partial C$ (Figure 1). Clearly the complement in $S^{n+2}$ of the configuration of Figure 1 is diffeomorphic to $X_{2}$. 


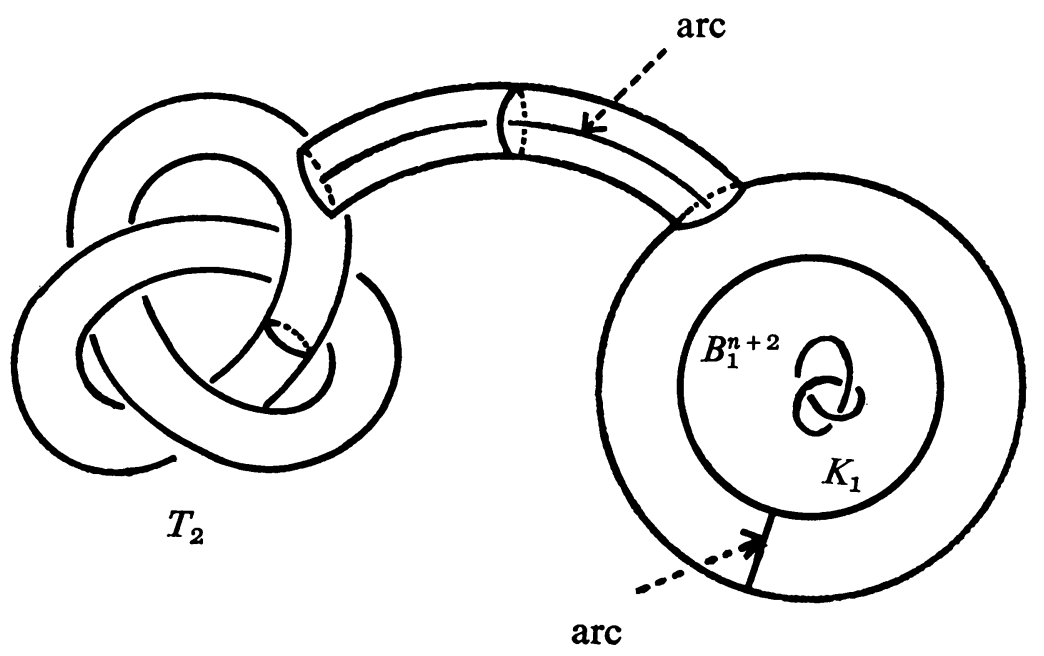

Figure 1

We also have that

$$
B_{1}^{n+2}-K_{1} \simeq X_{1} \vee S^{n+1}
$$

This homotopy equivalence is obtained in exactly the same way as the previous one, collapsing away from one boundary component of $\partial\left(B_{1}^{n+2}-T_{1}\right)$ and isolating the other in a wedge product decomposition.

Hence if $L$ is splittable, then $X=S^{n+2}-L \simeq X_{1} \vee X_{2} \vee S^{n+1}$. Let $*$ denote the base point of the wedge product. Then (up to homotopy equivalence) $\tilde{X}$ consists of one copy of $\tilde{X}_{1}$ and one copy of $\tilde{X}_{2}$, identified along $\tilde{*}$, the points lying over $*$, together with $Z$ copies of $S^{n+1}$, one stuck on at each point of identification of $\tilde{X}_{1}$ and $\tilde{X}_{2}$. Split $\tilde{X}$ into two pieces, $W_{1}=\tilde{X}_{1} \cup_{\tilde{*}} \bigcup_{i \in Z} S_{i}^{n+1}$ and $W_{2}=\tilde{X}_{2}$. Then $\tilde{X}$ $=W_{1} \cup_{\mathfrak{i k}} W_{2}$. The Mayer-Vietoris sequence for the triad $\left(\tilde{X}, W_{1}, W_{2}\right)$ yields $\cdots \rightarrow H_{i}(\tilde{*}) \rightarrow H_{i}\left(W_{1}\right) \oplus H_{i}\left(W_{2}\right) \rightarrow H_{i}(\tilde{X}) \rightarrow \cdots$. Hence $H_{i}(\tilde{X}) \cong H_{i}\left(\tilde{X}_{1}\right) \oplus H_{i}\left(\tilde{X}_{2}\right)$ for $2 \leqq i \leqq n$. With augmented homology \#

$$
0 \rightarrow H_{1}\left(W_{1}\right) \oplus H_{1}\left(W_{2}\right) \rightarrow H_{1}(\tilde{X}) \rightarrow H_{0}^{\#}(\tilde{*}) \rightarrow 0 .
$$

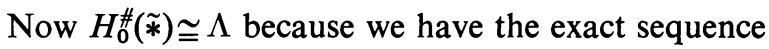

$$
0 \longrightarrow \stackrel{t-1}{\longrightarrow} \Lambda \stackrel{\varepsilon}{\longrightarrow} Z \longrightarrow 0,
$$

where $C_{0}(\tilde{*}) \cong \Lambda$ and $\varepsilon$ is the augmentation map. So (1) splits, and $H_{1}(\tilde{X}) \cong{ }_{\Lambda} H_{1}\left(\tilde{X}_{1}\right)$ $\oplus H_{1}\left(\tilde{X}_{2}\right) \oplus \Lambda$. Likewise, $H_{n+1}\left(W_{1}\right) \cong{ }_{\Lambda} H_{n+1}\left(\tilde{X}_{1}\right) \oplus \Lambda$. So

$$
H_{n+1}(\tilde{X}) \cong{ }_{\Lambda} H_{n+1}\left(W_{1}\right) \oplus H_{n+1}\left(W_{2}\right) \cong{ }_{\Lambda} H_{n+1}\left(\tilde{X}_{1}\right) \oplus H_{n+1}\left(\tilde{X}_{2}\right) \oplus \Lambda .
$$

This completes the proof of Theorem 4.1.

Let $L$ be an $n$-link of multiplicity $\mu$ which is geometrically $k$-splittable. Let $L_{i}$, $1 \leqq i \leqq k+1$, be the nonsplittable components of $L$. Let $X=S^{n+2}-L, X_{i}=S^{n+2}-L_{i}$, 
and $\tilde{X}_{i}$ denote the infinite cyclic cover of $X_{i}, 1 \leqq i \leqq k+1$. As a corollary to the proof of Theorem 4.1, we have

COROLlARY 4.2. If $L$ as above is geometrically $k$-splittable, then

$$
\begin{aligned}
H_{j}(\tilde{X} ; Z) & \cong \Lambda \bigoplus_{i=1}^{k+1} H_{j}\left(\tilde{X}_{i} ; Z\right), \quad 2 \leqq j \leqq n, \\
H_{1}(\tilde{X} ; Z) & \cong \Lambda \bigoplus_{i=1}^{k+1} H_{1}\left(\tilde{X}_{i} ; Z\right) \oplus k \Lambda, \\
H_{n+1}(\tilde{X} ; Z) & \cong \Lambda \bigoplus_{i=1}^{k+1} H_{n+1}\left(\tilde{X}_{i} ; Z\right) \oplus k \Lambda .
\end{aligned}
$$

COROLlaRY 4.3. If $L$ is an n-link of multiplicity $\mu$ which is $k$-splittable then $\Delta_{i}^{1}=0$, $1 \leqq i \leqq k$.

Let $P$ be a linear graph in $S^{3}$ which is $k$-splittable, and let $P_{i}, 1 \leqq i \leqq k+1$, be the nonsplittable components of $P$. As before let $X=S^{3}-P, X_{i}=S^{3}-P_{i}$, and $\tilde{X}_{i}$ denote the infinite cyclic cover of $X_{i}$. Let $G=\Pi_{1}(X)$ and $G_{i}=\Pi_{1}\left(X_{i}\right)$. Then

$$
G=G_{1} * G_{2} * \cdots * G_{k+1}
$$

and $\exists$ a natural monomorphism $\psi_{i}: G_{i} \rightarrow G, 1 \leqq i \leqq k+1$. Suppose that $\varphi: G \rightarrow J(t)$ is an epimorphism such that $\varphi_{i}=\varphi \psi_{i}: G_{i} \rightarrow J(t)$ is an epimorphism $1 \leqq i \leqq k+1$. Let $G^{*}=\operatorname{Ker} \varphi$ and $\tilde{X}$ be the infinite cyclic covering space of $X$ associated with $G^{*}$. Likewise, let $G_{i}^{*}$ be the kernel of $\varphi_{i}$, and $\tilde{X}_{i}$ the infinite cyclic cover of $X_{i}$ associated with $G_{i}^{*}$.

THEOREM 4.4 .

$$
\begin{aligned}
& H_{1}\left(G^{*} ; Z\right) \cong \Lambda \bigoplus_{i=1}^{k+1} H_{1}\left(G_{i}^{*} ; Z\right) \oplus k \Lambda, \\
& H_{2}\left(G^{*} ; Z\right) \cong \Lambda \bigoplus_{i=:}^{k+1} H_{2}\left(G_{i}^{*} ; Z\right) \text { (free abelian), } \\
& H_{p}\left(G^{*} ; Z\right)=0, \quad p \geqq 3 .
\end{aligned}
$$

Proof. By the proof to Theorem 4.1, we know that $X \simeq\left[\bigvee_{i=1}^{k+1} X_{i}\right] \vee\left[\bigvee_{j=1}^{k} S_{j}^{2}\right]$ and that $\tilde{X}$ can be constructed from the $\tilde{X}_{i}$ by identifying lifts of the wedge point $*$ at equivalent levels in the various $\tilde{X}_{i}$ and then tacking on a copy of $\left[\bigvee_{j=1}^{k} S_{j}^{2}\right]$ to each such vertex. For the purposes of calculating $H_{p}\left(G^{*} ; Z\right)$, the sphere packet $\left[\bigvee_{j=1}^{k} S_{j}^{2}\right]$ is irrelevant, so delete it, and consider $X=\bigvee_{i=1}^{k+1} X_{i}$. Now by [18], each $X_{i}$ is aspherical, and $\tilde{X}_{i} \cap \tilde{X}_{j} \subset \tilde{X}$ is aspherical, so $\tilde{X}$ will also be aspherical. This can be seen by considering the universal cover $\tilde{X} \sim \stackrel{p}{\rightarrow} \tilde{X}$ of $\tilde{X}$. Now $P^{-1}\left(\tilde{X}_{i}\right)$ will be the disjoint union of lots of copies of $\tilde{X}_{i} \tilde{X}$, the universal cover of $\tilde{X}_{i}$, since $\Pi_{1}\left(\tilde{X}_{i}\right) \stackrel{i^{\bullet}}{\rightarrow} \Pi_{1}(\tilde{X})$ is injective. The Mayer-Vietoris sequence in $\tilde{X} \sim$ shows that it aspherical. Hence $\tilde{X}=K\left(G^{*}, 1\right)$ and the theorem follows.

Let $\chi_{i}$ denote the Euler characteristic of $P_{i}$. Let $\left\{{ }_{i} \Delta_{k}^{1}\right\}$ denote the integral invariants of $H_{1}\left(\tilde{X}_{i} ; Z\right), i=1,2, \ldots, k+1$. 
Corollary 4.5. $H_{2}\left(G^{*} ; Z\right)=0$ iff ${ }_{i} \Delta_{1-x_{i}}^{1}(t) \neq 0$ for $1 \leqq i \leqq m$.

Proof. Corollary 2.8 and Theorem 4.4 yield this result.

Examples of isotopy linking (nonsplittability) [1], [21], [23] and homotopy linking [2], [8] of codimension 2 spheres are well known, the former usually being demonstrated by studying $\Pi_{1}$ and the latter by proving that an unknotted sphere is not homotopic to 0 in the complement of the other sphere. We will now exhibit two examples of 2-links of multiplicity 2 in $S^{4}$ which are not geometrically splittable and detectable as such by considering their homology invariants.

EXAmPLE 1. Spun link. In $R^{4}$ we take coordinates $x_{1}, x_{2}, x_{3}, x_{4}$. Let $R_{+}^{3}$ be the half-space described by $x_{4}=0$ and $x_{3} \geqq 0$ and $R_{0}^{2}$ the plane defined by $x_{4}=0$ and $x_{3}=0$. We consider two disjoint arcs in $R_{+}^{3}$ with endpoints in $R_{0}^{2}$ as shown in Figure 2.

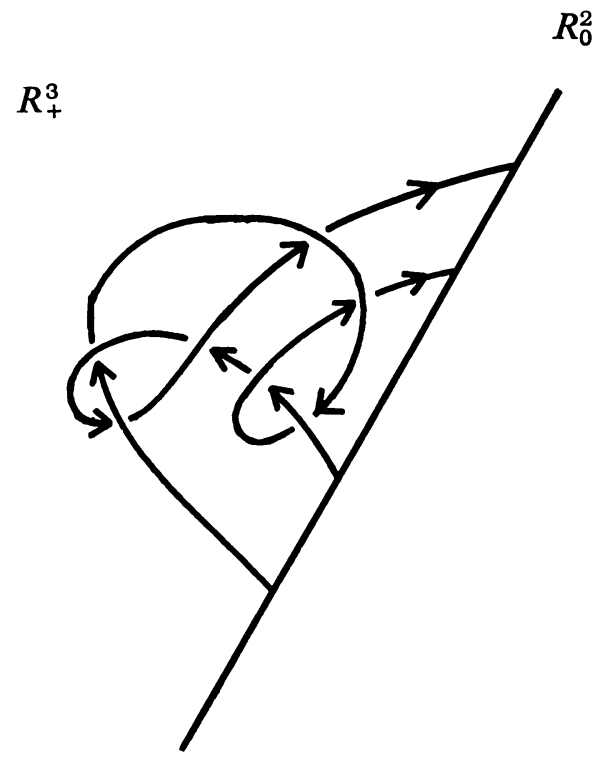

Figure 2

Following Artin [1] we rotate $R_{+}^{3}$ about $R_{0}^{2}$. Then the rotation of two arcs sweeps out two disjoint 2-spheres in $R^{4}$ and we obtain a 2-link $L$ of multiplicity 2 . By using Artin's theorem [1] it is easy to show that $\Pi_{1}\left(S^{4}-L\right)$ is isomorphic to the fundamental group of the complement of the linear graph shown in Figure 2 of [9]. Therefore, if $\tilde{X}$ is the infinite cyclic covering space of $X$ defined by an epimorphism $\varphi$ given by $(3.1)$, then $\left[t^{2}+t+1,2\right]$ is a presentation matrix for $H_{1}(\tilde{X} ; Z)$. Hence $L$ is not geometrically splittable by Theorem 4.1 .

EXAMPLE 2. Van Kampen's link of unknotted spheres [21], [23]. This time we spin the two arcs of Figure 3 to produce a 2-link of multiplicity 2, in which each of the components of the link is an unknotted $S^{2}$. 


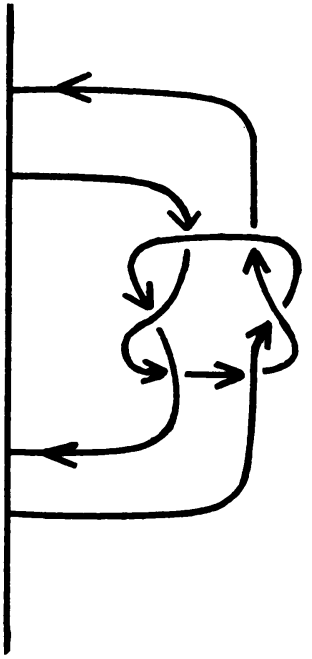

Figure 3

This time we obtain as a presentation matrix for $H_{1}(\tilde{X} ; Z)$ the $(1 \times 2)$ matrix $\left(t^{2}-t+1,0\right)$ so the link is clearly nonsplittable.

Related examples of nonsplittable $n$-links can be produced by surgery or by $(n-1)$-spinning [3], [8], [19] the configurations in Figures 2 and 3.

5. Finite cyclic coverings. For a classical knot $k: S^{1} \rightarrow S^{3}$ it is well known that if $A$ is a Seifert matrix for $k$, then $A+A^{\prime}$ is a presentation matrix for the 2-fold cyclic covering space of $S^{3}$ branched along $k$. We will obtain a similar result for $q$-simple $n$-links.

As before, let $L$ be an $n$-link of multiplicity $\mu$ and $X=S^{n+2}-L$.

Definition. $L$ is called $q$-simple $(q \geqq 0)$ if $\Pi_{i}(X)=\Pi_{i}\left(C_{\mu, n}\right)$ for $i \leqq q$, where $C_{\mu, n}=\left(\bigvee_{i=1}^{\mu} S_{i}^{1}\right) \vee\left(\bigvee_{j=1}^{\mu-1} S_{j}^{n+1}\right)$. Note that every link is 0 -simple. The trivial link is $q$-simple for all $q$. As is shown in [19], it is possible for an $n$-link to be $(n-1)$ simple and still be nontrivial.

Let $\tilde{X}$ denote the infinite cyclic cover of $X$ associated with $\operatorname{Kernel} \varphi$, where $\varphi$ is given by (3.1).

LEMMA 5.1. If an $n$-link $L$ of multiplicity $\mu$ is $q$-simple $q \geqq 1$ then

(i) $H_{1}(\tilde{X} ; Z) \cong \Lambda(\mu-1) \Lambda$,

(ii) if $2 \leqq q \leqq n$ then $H_{i}(\tilde{X} ; Z)=0,2 \leqq i \leqq q$, and

(iii) if $q \geqq n+1$ then $H_{n+1}(\tilde{X} ; Z) \cong(\mu-1) \Lambda$.

Proof. Consider the map $f: C_{\mu}=\bigvee_{j=1}^{\mu} S_{j}^{1} \rightarrow X$ which induces an isomorphism on $\Pi_{1}$. Let $M$ be the mapping cylinder of $f . M \simeq X$, and $\Pi_{1}\left(M, C_{\mu}\right)=0$ and $\Pi_{i}\left(M, C_{\mu}\right)=\Pi_{i}(X), i \geqq 2$. Let $\tilde{M}$ be the infinite cyclic cover of $M$ associated with the epimorphism $\varphi: \Pi_{1}(X) \rightarrow J(t)$. We then have induced infinite cyclic covers $\tilde{X}$ of $X$ and $\tilde{C}_{\mu}$ of $C_{\mu}$. Moreover, $H_{1}\left(\tilde{C}_{\mu} ; Z\right) \cong{ }_{\Lambda}(\mu-1) \Lambda$ and $H_{i}\left(\tilde{C}_{\mu} ; Z\right)=0, i \geqq 2$. 
Moreover, the inclusion $i_{*}: \Pi_{1}\left(\widetilde{C}_{\mu}\right) \cong \Pi_{1}(\tilde{X})$ is an isomorphism so

$$
i_{*}: H_{1}\left(\tilde{C}_{\mu}\right) \stackrel{\cong}{\longrightarrow} H_{1}(\tilde{X})
$$

and (i) is proved. If $2 \leqq q \leqq n$ then $\Pi_{i}\left(\tilde{M} ; \tilde{C}_{\mu}\right)=0$ for $i \leqq q$ and so $H_{i}\left(\tilde{M}, \widetilde{C}_{\mu}\right)=0$, $i \leqq q$, and (ii) is proved.

If $q \geqq n+1$, then consider $M$ to be the mapping cylinder of the map $f: C_{\mu, n} \rightarrow X$ which induces isomorphism on $\Pi_{i}, i \leqq q$. We have from the following Hurewicz exact ladder (substituting $\tilde{X}$ for $\tilde{M}$ )

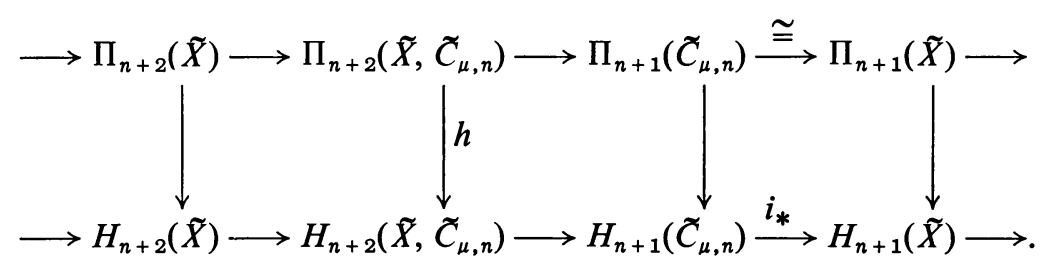

Since $h$ is an epimorphism then $i_{*}$ is an isomorphism and (iii) is proved.

THEOREM 5.2. Suppose that $L$ is a q-simple n-link of multiplicity $\mu$. Let $X_{k}^{u}$ be the $k$-fold unbranched cyclic covering space of $X=S^{n+2}-L$. Then

(i) $H_{1}\left(X_{k}^{u} ; Z\right) \cong Z \operatorname{Cok}\left(t^{k}-1\right) \oplus Z$ where $\left(t^{k}-1\right): H_{1}(\tilde{X} ; Z) \rightarrow H_{1}(\tilde{X} ; Z)$,

(ii) if $q \geqq 1$ then $H_{1}\left(X_{k}^{u} ; Z\right) \cong[k(\mu-1)+1] Z$,

(iii) if $2 \leqq q \leqq n$ then $H_{i}\left(X_{k}^{u} ; Z\right)=0,2 \leqq i \leqq q$,

(iv) if $1 \leqq q \leqq n$ then $H_{q+1}\left(X_{k}^{u} ; Z\right) \cong \operatorname{Cok}\left(t^{k}-1\right)$ where $\left(t^{k}-1\right): H_{q+1}(\tilde{X} ; Z)$ $\rightarrow H_{q+1}(\tilde{X} ; Z)$,

(v) if $q \geqq n+1$ then $H_{n+1}\left(X_{k}^{u} ; Z\right) \cong k(\mu-1) Z$.

Proof. The short exact sequence of chain complexes

$$
0 \longrightarrow C_{*}(\tilde{X} ; Z) \stackrel{\left(t^{k}-1\right)}{\longrightarrow} C_{*}(\tilde{X} ; Z) \longrightarrow C_{*}\left(X_{k}^{u} ; Z\right) \longrightarrow 0
$$

induces the long exact sequence of homolngy

$$
\cdots \longrightarrow H_{i}(\tilde{X} ; Z) \stackrel{\left(t^{k}-1\right)}{\longrightarrow} H_{i}(\tilde{X} ; Z) \longrightarrow H_{i}\left(X_{k}^{u} ; Z\right) \longrightarrow \cdots
$$

This gives us

$$
H_{1}(\tilde{X} ; Z) \stackrel{\left(t^{k}-1\right)}{\longrightarrow} H_{1}(\tilde{X} ; Z) \longrightarrow H_{1}\left(X_{k}^{u} ; Z\right) \longrightarrow
$$

from which (i) immediately follows.

Now if $q \geqq 1$, then we have

$$
0 \longrightarrow(\mu-1) \Lambda \stackrel{\left(t^{k}-1\right)}{\longrightarrow}(\mu-1) \Lambda \longrightarrow H_{1}\left(X_{k}^{u} ; Z\right) \longrightarrow
$$


which yields

$$
0 \rightarrow k(\mu-1) Z \rightarrow H_{1}\left(X_{k}^{u} ; Z\right) \rightarrow Z \rightarrow 0
$$

and so (ii) is proved. Since $H_{i}(\tilde{X} ; Z)=0$ for $2 \leqq i \leqq q$ the exact sequence yields immediately (iii) and (iv) when $q \geqq 2$. If $q=1$, then $\left(t^{k}-1\right): H_{1}(\tilde{X} ; Z) \rightarrow H_{1}(\tilde{X} ; Z)$ is injective and (iv) is proved. The proof for (v) is similar to that of (ii) and left to the reader.

Suppose now that $M(t)$ is a $\Lambda$-presentation matrix for $H_{q+1}(\tilde{X} ; Z)$, and that $M(t)$ has $m$ columns. Let $\left[t^{k}-1\right]$ denote the $m \times m$ diagonal matrix with $t^{k}-1$ down the diagonal, and

$$
\left(\begin{array}{c}
M \\
{\left[t^{k}-1\right]}
\end{array}\right)
$$

the matrix obtained from $M(t)$ by adjoining $\left[t^{k}-1\right]$ to the bottom.

COROLLARY 5.3. With the hypotheses of Theorem 5.2, we have

(i) if $q \geqq 1$ then

$$
\left(\begin{array}{c}
M(t) \\
{\left[t^{k}-1\right]}
\end{array}\right)
$$

is a presentation matrix for $H_{q+1}\left(X_{k}^{u} ; Z\right)$ as a $\Lambda$-module,

(ii) if $1 \leqq q \leqq n$ and $k=2$ then $M(-1)$ is a presentation matrix for $H_{q+1}\left(X_{2}^{u} ; Z\right)$ as an abelian group,

(iii) if $q \geqq 0, k=2$ and $\mu=1$ then

$$
\left(\begin{array}{c|c} 
& 0 \\
M(-1) & \vdots \\
& 0 \\
\hline 0 \cdots 0 & 0
\end{array}\right)
$$

is a presentation matrix for $H_{1}\left(X_{2}^{u} ; Z\right)$ as an abelian group.

Proof. (i) follows immediately from Lemma 8 of [3]. Now if $k=2$, then $\left(t^{2}-1\right)$ $=(t-1)(t+1)$ as a composite of homomorphisms. If $1 \leqq q<n+1$, then the arguments of $\S 2$ apply to show

$$
H_{q+1}(\tilde{X} ; Z) \stackrel{(t-1)}{\cong} H_{q+1}(\tilde{X} ; Z) .
$$

So Cok $\left(t^{2}-1\right) \cong{ }_{\Lambda} \operatorname{Cok}(t+1)$, and $\operatorname{Cok}(t+1)$ is presented as a $\Lambda$-module by the matrix

$$
\left(\begin{array}{c}
M(t) \\
{[t+1]}
\end{array}\right)
$$


hence as an abelian group by the matrix $(M(-1)$ ). If $k=2$ and $\mu=1$ (we have a knot in this case), then again the arguments of $\$ 2$ yield

$$
H_{1}(\tilde{X} ; Z) \stackrel{(t-1)}{\cong} H_{1}(\tilde{X} ; Z)
$$

and (iii) is proved.

A more interesting situation to study is that of the branched cyclic covering.

THEOREM 5.4. Let $L$ be an n-link of multiplicity $\mu$, and $X_{k}^{u}$ and $X_{k}^{b}$ denote the $k$-fold unbranched and branched cyclic covers, respectively. Then

(i) $0 \longrightarrow \mu Z \longrightarrow H_{1}\left(X_{k}^{u} ; Z\right) \stackrel{i_{*}}{\longrightarrow} H_{1}\left(X_{k}^{b} ; Z\right) \longrightarrow 0$ is exact, and is split exact if either $L$ is completely splittable or if $L$ is 1-simple,

(ii) $i_{*}: H_{i}\left(X_{k}^{u} ; Z\right) \cong H_{i}\left(X_{k}^{b} ; Z\right)$ if $2 \leqq i \leqq n$,

(iii) $0 \longrightarrow(\mu-1) Z \longrightarrow H_{n+1}\left(X_{k}^{u} ; Z\right) \stackrel{i_{\bullet}}{\longrightarrow} H_{n+1}\left(X_{k}^{b} ; Z\right) \longrightarrow 0$ is exact, and is split exact if $L$ is completely splittable, or if $L$ is $(n+1)$-simple.

Proof. By excision,

$$
\begin{aligned}
H_{i}\left(X_{k}^{b}, X_{k}^{u} ; Z\right) & \cong \mu Z, & & i=2, n+2, \\
& \cong 0, & & \text { otherwise. }
\end{aligned}
$$

Now from the exact sequence of the pair we have

$$
\begin{gathered}
\longrightarrow H_{2}\left(X_{k}^{b}\right) \longrightarrow H_{2}\left(X_{k}^{b}, X_{k}^{u}\right) \stackrel{\partial}{\longrightarrow} H_{1}\left(X_{k}^{u}\right) \longrightarrow H_{1}\left(X_{k}^{b}\right) \longrightarrow 0 \\
\mu Z
\end{gathered}
$$

Now $\partial$ is injective, as is easily seen by considering the pair $\left(X_{k}^{u}, \partial X_{k}^{u}\right)$ covering the pair $(X, \partial X)$. Let $p:\left(X_{k}^{u}, \partial X_{k}^{u}\right) \rightarrow(X, \partial X)$ be the covering map. Then we have the commutative diagram

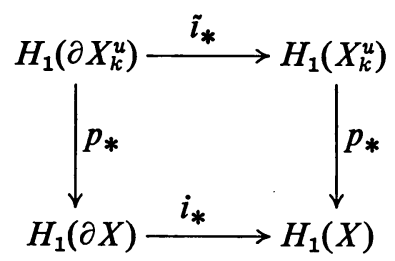

Now if $n>1$, then $H_{1}\left(\partial X_{k}^{u}\right) \cong \mu Z \cong H_{1}(\partial X)$ and $p_{*}: H_{1}\left(\partial X_{k}^{u}\right) \rightarrow H_{1}(\partial X)$ is multiplication by $k$, hence injective. Now $i_{*}: H_{1}(\partial X) \rightarrow H_{1}(X)$ is an isomorphism in this case, so $\tilde{i}_{*}$ is injective. Clearly then $\partial$ is injective. If $n=1$, the above argument goes through by considering the direct summand of $H_{1}(\partial X)$ generated by the meridian curves.

Now Crowell [5] proves that for a knot $(\mu=1)$ then $0 \rightarrow Z \rightarrow H_{1}\left(X_{k}^{u}\right) \rightarrow H_{1}\left(X_{k}^{b}\right)$ $\rightarrow 0$ splits. As in $\S 4$, let $\left\{L_{i}\right\}_{i=1}^{p}$ be the nonsplittable components of $L$, and $X_{i}$ $=S^{n+2}-L_{i}$, and $X_{k, i}^{u}$ and $X_{k, i}^{b}$ denote the $k$-fold unbranched and branched cyclic 
covers, respectively, of $X_{i}$. Then $X \simeq\left(\bigvee_{j=1}^{p-1} S_{j}^{n+1}\right) \vee\left\{\bigvee_{i=1}^{p} X_{i}\right\}$. Let $S=\bigvee_{j=1}^{p-1} S_{j}^{n+1}$. Then $X_{k}^{u} \simeq\left(\bigcup X_{k, i}^{u}\right) \cup \tilde{*}\left(\bigcup_{j=0}^{k-1} S_{j}\right)$, that is the union of one copy each of $X_{k, i}^{u}$ and $k$ copies $\left\{S_{j}\right\}_{j=0}^{k=1}$ of $S$, identified along $k$ points $\tilde{*}$, the lift of the base point $*$ in the wedge product decomposition for $X$. Now the Mayer-Vietoris sequence for $X_{k}^{u}$ gives us

(a) $H_{i}\left(X_{k}^{u}\right) \cong \bigoplus_{i=1}^{p} H_{i}\left(X_{k, i}^{u}\right)$ for $2 \leqq i \leqq n$, and

(b) $0 \rightarrow \bigoplus_{i=1}^{p} H_{1}\left(X_{k, i}^{u}\right) \rightarrow H_{1}\left(X_{k}^{u}\right) \rightarrow Z \rightarrow 0$, and

(c) $H_{n+1}\left(X_{k}^{u}\right)=\bigoplus_{i=1}^{p} H_{n+1}\left(X_{k, i}^{u}\right) \oplus k(p-1) Z$.

Likewise, we get (a) and (b) relating the homology of $X_{k}^{b}$ and $\left\{X_{k, i}^{b}\right\}$. Moreover we have that the inclusion $i: X_{k}^{u} \rightarrow X_{k}^{b}$ induces the following exact diagram, when the link $L$ is completely splittable:

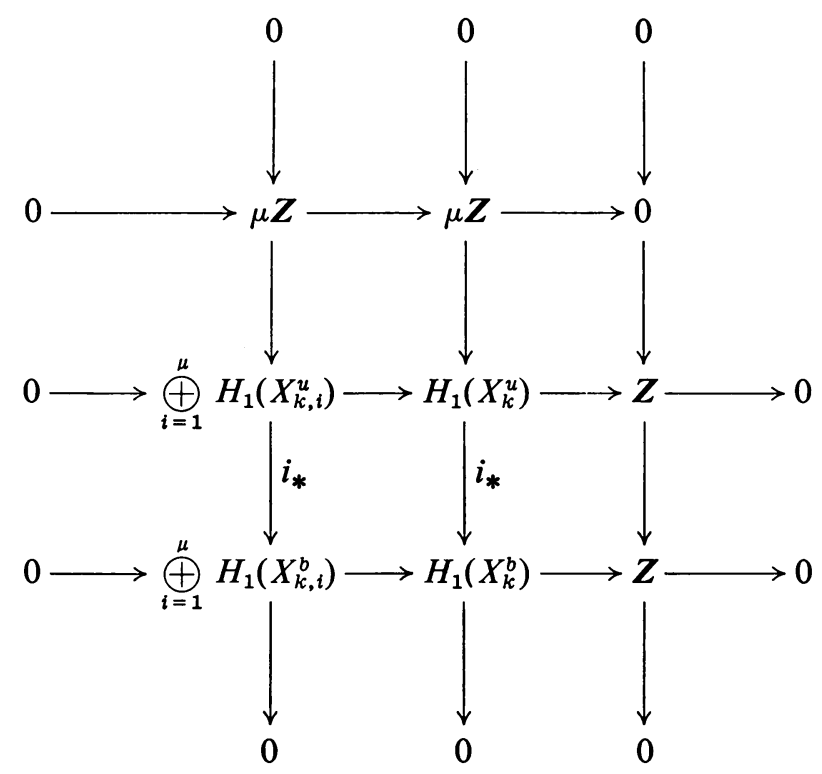

The left-hand column is the direct sum of $\mu$ split exact sequences, so the middle column splits.

If $L$ is 1 -simple, then as in the proof of Lemma 5.1 we have the map $i: C_{\mu} \rightarrow X$ inducing an isomorphism on $\Pi_{1}$, and if $M$ is the mapping cylinder of $i$, and $M_{k}$ the $k$-fold cover of $M$, then we have the $k$-fold cover $C_{\mu, k}$ of $C_{\mu}$ induced. Moreover,

$$
H_{1}\left(C_{\mu, k}\right) \stackrel{i_{*}}{\cong} H_{1}\left(M_{k}\right) \cong H_{1}\left(X_{k}^{u}\right)
$$

and the elements of the bouquet $C_{\mu}$ lift to $C_{\mu, k}$ to generate $\mu Z$ as a direct summand of $H_{1}\left(X_{k}^{u}\right)$. This copy of $\mu Z$ is exactly that generated by the meridians in $\partial X_{g}^{u}$, so (i) is proved. 
Now $X_{k}^{b}$ is an orientable $(n+2)$-manifold, and we have from the exact sequence of the pair $\left(X_{k}^{b}, X_{k}^{u}\right)$,

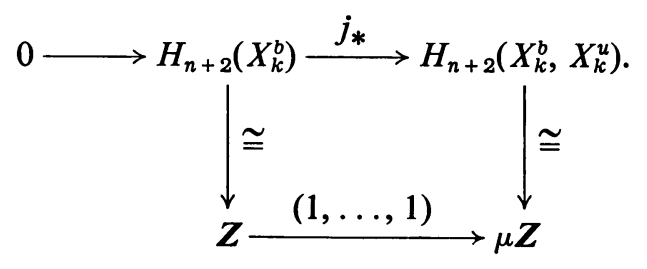

That is, the map $j_{*}$ can be represented by the $(1 \times \mu)$ matrix $(1, \ldots, 1)$. Hence

$$
0 \longrightarrow(\mu-1) Z \stackrel{\partial}{\longrightarrow} H_{n+1}\left(X_{k}^{u}\right) \stackrel{i_{*}}{\longrightarrow} H_{n+1}\left(X_{k}^{b}\right) \longrightarrow 0
$$

is exact. Moreover, if $L$ is completely splittable, then each of the splitting spheres $\left\{S_{j}^{n+1}\right\}$ in $X$ lifts to a splitting sphere in $X_{k}^{u}$, so as in $\S 4$ we can isolate $\bigvee_{j=1}^{\mu-1} S_{j}^{n+1}$ in a wedge product decomposition for $X_{k}^{u}$. It is also clear that the direct summand $(\mu-1) Z$ of $H_{n+1}\left(X_{k}^{u}\right)$ from the wedge product decomposition is precisely Im $\partial$ in (1) above.

If $L$ is $(n+1)$-simple, the argument follows that of (i) above and is left to the reader. This completes the proof of Theorem 5.4.

Note that the sequence $0 \rightarrow \mu Z \rightarrow H_{1}\left(X_{k}^{u} ; Z\right) \rightarrow H_{1}\left(X_{k}^{b}\right) \rightarrow 0$ can fail to be split exact if $L$ is not completely splittable. We have the following example for $n=1$, $\mu=2, k=2$ due to S. Kinoshita:

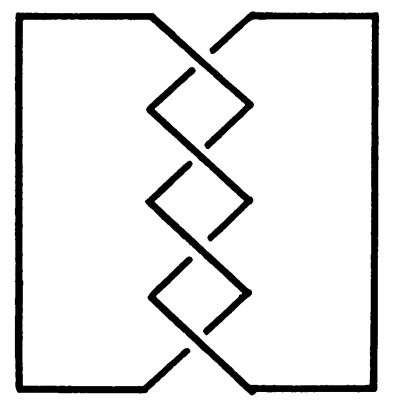

FIGURE 4

For the link of Figure 4, we have

$$
\begin{aligned}
& 0 \longrightarrow Z \oplus Z \longrightarrow H_{1}\left(X_{2}^{u}\right) \longrightarrow H_{1}\left(X_{2}^{b}\right) \longrightarrow 0 \\
& \text { ॥2 } \quad 112 \\
& Z \oplus Z \oplus Z_{2} \quad Z_{4}
\end{aligned}
$$

which does not split.

Combining previous results, we have

COROLlary 5.5. L a q-simple n-link. Then 
(i) If $1 \leqq q \leqq n-1$ then $H_{1}\left(X_{k}^{b} ; Z\right)=[(k-1)(\mu-1)] Z, H_{i}\left(X_{k}^{b} ; Z\right)=0,2 \leqq i \leqq q$, and $H_{q+1}\left(X_{k}^{b} ; Z\right) \cong H_{q+1}\left(X_{k}^{u} ; Z\right) \cong \operatorname{Cok}\left(t^{k}-1\right)$ where $\left(t^{k}-1\right): H_{q+1}(\tilde{X} ; Z) \rightarrow H_{q+1}(\tilde{X} ; Z)$.

(ii) If $1 \leqq q \leqq n-1, k=2$ and $M(t)$ presents $H_{q+1}(\tilde{X} ; Z)$ as a $\Lambda$-module then $M(-1)$ presents $H_{q+1}\left(X_{2}^{b} ; Z\right)$ as an abelian group.

COROllary 5.6. Suppose that $L$ is a q-simple $(2 q+1)$-link of multiplicity $\mu$, where $q \geqq 1$ if $\mu \geqq 2$. If $A$ is a Seifert matrix for $L$, then $A+(-1)^{q} A^{\prime}$ is a presentation matrix for $H_{q+1}\left(X_{2}^{b} ; Z\right)$.

\section{REFERENCES}

1. E. Artin, Zur Isotopies zweidimensionaler Flächen im $R_{4}$, Abh. Math. Sem. Univ. Hamburg 4 (1925), 174-177.

2. J. J. Andrews and M. L. Curtis, Knotted 2-spheres in the 4-sphere, Ann. of Math. (2) 70 (1959), 565-571. MR 21 \#5964.

3. J. J. Andrews and D. W. Sumners, On higher-dimensional fibered knots, Trans. Amer. Math. Soc. 153 (1971), 415-426.

4. R. C. Blanchfield, Intersection theory of manifolds with operators with applications to knot theory, Ann. of Math. (2) 65 (1957), 340-356. MR 19, 53.

5. R. H. Crowell, $H_{2}$ of subgroups of knot groups, Illinois J. Math. 14 (1970), 665-673.

6. R. H. Crowell and D. S. Cochran, $H_{2}\left(G^{\prime}\right)$ for tamely embedded graphs, Quart. J. Math. Oxford Ser. (2) 21 (1970), 25-27. MR 41 \#2660.

7. R. H. Crowell and R. H. Fox, Introduction to knot theory, Ginn, Boston, Mass., 1963. MR 26 \#4348.

8. D. B. A. Epstein, Linking spheres, Proc. Cambridge Philos. Soc. 56 (1960), 215-219. MR 22 \#8514.

9. S. Kinoshita, Alexander polynomials as isotopy invariants. I, Osaka. Math. J. 10 (1958), 263-271. MR 21 \#1605.

10. - Elementary ideals of linear graphs in a 3-sphere, Notices Amer. Math. Soc. 14 (1967), 676. Abstract \#648-154.

11. J. Levine, A characterization of knot polynomials, Topology 4 (1965), 135-141. MR 31 \#5194.

12. - Knot cobordism groups in codimension two, Comment. Math. Helv. 44 (1969), 229-244. MR 39 \#7618.

13. - Polynomial invariants of knots of codimension two, Ann. of Math. (2) 84 (1966), 537-554. MR 34 \#808.

14. —_ Unknotting spheres in codimension two, Topology 4 (1965), 9-16. MR 31 \#4045.

15. J. W. Milnor, Infinite cyclic coverings, Conference on the Topology of Manifolds (Michigan State University, E. Lansing, Mich., 1967), Prindle, Weber and Schmidt, Boston, Mass., 1968, pp. 115-133. MR 39 \#3497.

16. K. Murasugi, On a certain numerical invariant of link types, Trans. Amer. Math. Soc. 117 (1965), 387-422. MR 30 \#1506.

17. L. P. Neuwirth, Knot groups, Ann. of Math. Studies, no. 56, Princeton Univ. Press, Princeton, N. J., 1965. MR 31 \#734.

18. C. D. Papakyriakopoulos, On Dehn's lemma and the asphericity of knots, Ann. of Math. (2) 66 (1957), 1-26. MR 19, 761.

19. D. W. Sumners, On an unlinking theorem, Proc. Cambridge Philos. Soc. (to appear).

20.,$- \mathrm{H}_{2}$ of the commutator subgroup of a knot group, Proc. Amer. Math. Soc. 28 (1971), 319-320. 
21. E. H. Van Kampen, Zur Isotopie zweidimensionaler Flächen im $R_{4}$, Abh. Math. Sem. Univ. Hamburg 6 (1927), 216.

22. H. Zassenhaus, The theory of groups, Chelsea, New York, 1958.

23. E. C. Zeeman, Linking spheres, Abh. Math. Sem. Univ. Hamburg 24 (1960), 149-153. MR $22 \# 8513$.

Department of Mathematics, University of Toronto, Toronto, Ontario, Canada

Department of Mathematics, Florida State University, Tallahassee, Florida 32306

Current address (Shinohara): Department of Mathematics, University of Georgia, Athens, Georgia 30601 